anslysis of atomic structure in terms of protons and neutrons, and the repercussion of those phenomena upon the law of conservation of energy, also came in for a fair amount of discussion. Fowler and Dirac (Cambridge), Tamm, Frenkel and others joined in this discussion; its general tone differed from that of the similar discussion which took place at the recent York meeting of the British Association in that the unexplained behaviour of the energy distribution in the $\beta$-ray emission of radioactive bodies did not appear to shake the confidence of the speakers in the utility of the postulate of the conservation of energy when applied to subatomic phonomena.

Victor Cofman.

\title{
Iron-cored Coils for Radio Frequencies
}

$\mathrm{F}^{\mathrm{o}}$ OR several years past iron-cored coils for oscillatory circuits have beon used by telephone engineers at audio frequencies and at the low radio frequencies which are used in carrier current telephony. In order to reduce the losses which would accompany the use of iron at frequencies of the order of 50,000 cycles per second, the magnetic material was made up in the form of iron dust or filings embedded in a wax or cement in such a way that each particle of iron was insulated from its neighbours. In this way the eddy currents were very restricted in their paths, and the resulting iron losses were reduced to a minimum, so that the presence of the iron increased the inductance of the coil to a much greater extent than its resistance. When attempts were made to use such cores for coils required for higher radio frequencies, however, they were found to be a disadvantage, and the best design of coil for use in the medium broadcasting band of frequencies has been found to be a single-layer air-cores solenoid of suitable proportions of length to diameter.

In the Wireless World of September 16 an announcement was made of the production in Germany of a new magnetic material named "Ferrocart", which may have a considerable influence on the design and construction of inductance coils for, at any rate, moderately high radio frequencies. Details as to the exact composition and the mode of manufacture of this material are not yet available, but paper laminations appear to be used to carry the dust core, and the result is a decided improvoment upon the old form of core with iron particles. Also a considerable advantage is obtained from the fact that "Ferrocart" can be made up in the form of annular rings for use in the toroidal type of inductance coil. In this manner the air path of the magnetic field of the coil is reduced to a minimum; and since there is less external stray field, the screening of coils in a receiver or other piece of apparatus is rendered an easier problem. In the "Ferrocart" coil the inductance is obtained with considerably fower turns than is necessary when an air core is used, so that it becomes possible to make the coils quite small while still retaining thick or multi-stranded wire to ensure low copper losses.

Later issuas of the Wireless World have contained the results of comparison tests of typical air-core coils with two coils of the new type, the larger of which was enclosed in a screening box about 2 inches long and 2 inches in diameter. At frequencies corresponding to the medium broadcast band of wave. lengths, each of the coils gave a considerably better performance than two typical commercial coils of greater dimensions, as used in modern broadcast receivers.

The better of the two "Ferrocart" coils was, indeed, only surpassed by an unusually well-designed air coil using stranded wire wound on a three-inch diameter former, containing two or three times the amount of copper wire and prosenting considerable difficulty in screening it without loss of efficiency. It is understood that a firm has already arranged to develop the applications of "Ferrocart" in Great Britain.

\section{Weather Charts of the Northern Hemisphere}

$\mathrm{T}$ HE Deutsche Scewarte, Hamburg, has undertaken to produce on behalf of the International Meteorological Organisation, daily synoptic weather charts covering the whole of the northern hemisphere. It is not intended to produce a synoptic chart differing from those published daily by tho British Meteorological Office merely in the size of the area dealt with-tho British charts cover only a small portion of the large area between the equator and the northern tropic-but one which shall contain the maximum possible amount of detail. For this purpose, it will be necessary to use more material than is provided by the ordinary international exchange of weather reports by wireless, and consequently these extended weather maps will not be available soon enough for direct use in daily short period weather forecasting. Their indirect value in forecasting may, on the other hand, be very great.

It is the view of many meteorologists that an understanding of the causes of long spells of abnormal woather can only be arrived at by the study of charts of this kind, a study which can be pursued at leisure, working on past weather situations. In the future such extended charts will be available in time for direct use in daily forecasting, but meanwhile the knowledge gained from the study of those now to bo prepared should prove valuable in intorpreting existing charts in much the same way that knowledge gained from a study of the synoptic weather charts of Europe and the North Atlantic solved some of the difficulties of forecasting from charts covering only a portion of Europe such as were used in most countries until recent years. But it is in the further development of long range forecasting that they art likely to be of the greatest utility. Seasonal forecasting of rainfall has been carried out with some measure of success in India and some other countries by means of equations dorived from the theory of correlation, the future rainfall being correlated with various antecedent values of meteorological elements in distant countries. It is possible that the new charts may cause this method to be replaced by other more scientific methods based on a better understanding of the general circulation of the atmosphere.

It is hoped to begin the series with the period

No. 3289, Vol. 130] 